Atherosclerosis in Systemic Diseases and in Metabolic Bone Diseases of the Musculoskeletal System, Division of Rheumatology, Hospital Universitario Marqués de Valdecilla, IDIVAL, Santander, Spain; ${ }^{2}$ López Albo' Post-Residency Programme, Hospital Universitario Marqués de Valdecilla, Santander, Spain; ${ }^{3}$ Hospital Universitario San Cecilio, Division of Pediatrics, Granada, Spain; ${ }^{4}$ Department of Epidemiology and Computational Biology, School of Medicine, University of Cantabria, and CIBER Epidemiología y Salud Pública (CIBERESP), IDIVAL, Santander, Spain; ${ }^{5}$ Hospital Universitario San Cecilio, Systemic Autoimmune Diseases Unit, Granada, Spain; ${ }^{6}$ Hospital Universitario Marqués de Valdecilla, Division of Pediatrics, Santander, Spain; ${ }^{7}$ Hospital Universitario Marqués de Valdecilla, Division of Nephrology, Santander, Spain; ${ }^{8}$ Hospital Universitario Lucus Augusti, Division of Rheumatology, Lugo, Spain; ${ }^{9}$ Hospital Universitario de Bellvitge, Division of Rheumatology, Barcelona, Spain; ${ }^{10}$ Hospital Universitario Central de Asturias, Division of Rheumatology, Oviedo, Spain; ${ }^{11}$ Hospital Universitario Severo Ochoa, Division of Rheumatology, Madrid, Spain; ${ }^{12}$ Hospital Regional Universitario Carlos Haya, Division of Rheumatology, Málaga, Spain; ${ }^{13}$ Hospital da Costa Burela, Division of Nephrology, Lugo, Spain; ${ }^{14}$ Hospital Universitario Lucus Augusti, Division of Nephrology, Lugo, Spain; ${ }^{15}$ Hospital Universitario de La Princesa, Dermatology Department, Madrid, Spain; ${ }^{16}$ Hospital Universitario Virgen del Rocío, Division of Rheumatology, Sevilla, Spain; ${ }^{17}$ Hospital Universitario de Basurto, Division of Rheumatology, Bilbao, Spain; ${ }^{18}$ Instituto de Parasitolog ía y Biomedicina 'LópezNeyra', CSIC, PTS Granada, Granada, Spain; ${ }^{19}$ Hospital Universitario de La Princesa, IIS-Princesa, Division of Rheumatology, Madrid, Spain; ${ }^{20}$ University of Cantabria, School of Medicine, Santander, Spain; ${ }^{21}$ University of the Witwatersrand, Cardiovascular Pathophysiology and Genomics Research Unit, School of Physiology, Faculty of Health Sciences, Johannesburg, South Africa

Background: $\lg \mathrm{A}$ vasculitis ( $\lg \mathrm{AV})$ and $\lg \mathrm{A}$ nephropathy $(\operatorname{Ig} \mathrm{AN})$ are inflammatory conditions that share pathogenic and molecular mechanisms [1] and may represent different outcomes of a continuous spectrum of disease [2]. Interleukin (IL)17A has been identified as a common genetic risk locus for several immune-mediated diseases [3, 4].

Objectives: To determine whether IgAV and IgAN exhibit a different IL17A association pattern.

Methods: Five IL17A tag polymorphisms (rs4711998, rs8193036, rs3819024, rs2275913 and rs7747909) were genotyped in 388 Caucasian patients with IgAV, 99 patients with IgAN and 1,003 sex and ethnically matched healthy controls.

Results: No statistically significant differences between patients with IgAV and healthy controls and between patients with IgAN and healthy controls were observed when each IL17A genetic variant was analyzed independently (Table 1). Similarly, IgAV patients exhibited similar genotype and allele IL17A frequencies than those with IgAN (Table 1). Moreover, no genotype or allele differences between IgAV patients who developed nephritis and patients with IgAN were detected. Furthermore, haplotype frequencies were similar in patients with $\lg A V$, IgAV and nephritis and those with $\lg A N$.

Table 1. Genotype and allele frequencies of IL17A gene in patients with IgA vasculitis, patients with IgA nephropathy and healthy controls.

\begin{tabular}{|c|c|c|c|c|c|c|c|}
\hline Polymorphism & Change & Data set & $1 / 1$ & $1 / 2$ & $2 / 2$ & 1 & 2 \\
\hline \multirow[t]{3}{*}{ rs4711998 } & $\mathrm{G} / \mathrm{A}$ & IgAV & 53.4 (207) & 38.9 (151) & $7.7(30)$ & 72.8 (565) & $27.2(211)$ \\
\hline & & IgAN & $49.0(48)$ & $42.9(42)$ & $8.2(8)$ & 70.4 (138) & $29.6(58)$ \\
\hline & & Controls & 52.7 (529) & $41.2(413)$ & $6.1(61)$ & 73.3 (1471) & 26.7 (535) \\
\hline \multirow[t]{3}{*}{ rs8193036 } & T/C & $\lg A \mathrm{~V}$ & 57.0 (221) & 38.4 (149) & $4.6(18)$ & 76.2 (591) & $23.8(185)$ \\
\hline & & IgAN & $64.3(63)$ & $31.6(31)$ & $4.1(4)$ & 80.1 (157) & 19.9 (39) \\
\hline & & Controls & 60.3 (605) & 35.2 (353) & $4.5(45)$ & 77.9 (1563) & 22.1 (443) \\
\hline \multirow[t]{3}{*}{ rs3819024 } & $A / G$ & IgAV & $44.1(171)$ & 43.3 (168) & $12.6(49)$ & 65.7 (510) & $34.3(266)$ \\
\hline & & IgAN & 39.4 (39) & $54.5(54)$ & $6.1(6)$ & 66.7 (132) & $33.3(66)$ \\
\hline & & Controls & 45.6 (457) & 44.6 (447) & 9.9 (99) & 67.8 (1361) & 32.2 (645) \\
\hline \multirow[t]{3}{*}{ rs2275913 } & $\mathrm{G} / \mathrm{A}$ & $\lg A \mathrm{~V}$ & 44.6 (172) & $43.3(167)$ & $12.2(47)$ & $66.2(511)$ & $33.8(261)$ \\
\hline & & IgAN & 39.8 (39) & $53.1(52)$ & $7.1(7)$ & $66.3(130)$ & $33.7(66)$ \\
\hline & & Controls & 44.8 (449) & $44.2(443)$ & 11.1 (111) & 66.8 (1341) & $33.2(665)$ \\
\hline \multirow[t]{3}{*}{ rs7747909 } & $\mathrm{G} / \mathrm{A}$ & $\lg A V$ & 53.9 (209) & 39.4 (153) & $6.7(26)$ & $73.6(571)$ & 26.4 (205) \\
\hline & & IgAN & 41.1 (39) & $54.7(52)$ & $4.2(4)$ & $68.4(130)$ & $31.6(60)$ \\
\hline & & Controls & $53.0(532)$ & 39.4 (395) & $7.6(76)$ & 72.7 (1459) & 27.3 (547) \\
\hline
\end{tabular}

Conclusion: Our results revealed that IgAV and IgAN share a similar IL17A association pattern.

REFERENCES:

[1] N Engl J Med 2013;368:2402-14.

[2] Am J Kidney Dis 1988;12:373-7.

[3] Ann Rheum Dis 2014;73:1742-5.

[4] Mediators Inflamm 2018;2018:1395823.

Acknowledgements: This study was supported by European Union FEDER funds and "Fondo de Investigaciones Sanitarias" (grant PI18/00042) from 'Instituto de Salud Carlos III' (ISCIII, Health Ministry, Spain). DP-P is a recipient of a Río Hortega programme fellowship from the ISCIII, co-funded by the European Social Fund (ESF, 'Investing in your future') [grant number CM20/00006];
SR-M is supported by funds of the RETICS Program co-funded by the European Regional Development Fund (ERDF) [grant number RD16/0012/0009]; VP-C is supported by a pre-doctoral grant from IDIVAL [grant number PREVAL 18/01]; BA-M is a recipient of a 'López Albo' Post-Residency Programme funded by Servicio Cántabro de Salud; LL-G is supported by funds of IDIVAL [grant numbe INNVAL20/06]; RL-M is a recipient of a Miguel Servet type I programme fellowship from the ISCIII, co-funded by the European Social Fund (ESF, 'Investing in your future') [grant number CP16/00033].

Disclosure of Interests: Diana Prieto-Peña: None declared, Fernanda Genre: None declared, Sara Remuzgo Martinez: None declared, Verónica Pulito-Cueto: None declared, Belén Atienza-Mateo: None declared, Belén Sevilla: None declared, Javier Llorca: None declared, Norberto Ortego: None declared, Leticia Lera-Gómez: None declared, Maite Leonardo: None declared, Ana Peñalba: None declared, Luis Martín-Penagos: None declared, Jose Alberto Miranda-Filloy: None declared, J. Narváez: None declared, LUIS CAMINAL MONTERO: None declared, PAZ COLLADO: None declared, Antonio Fernandez-Nebro: None declared, Gisela Díaz-Cordoves: None declared, Secundino Cigarrán: None declared, Jesús Calviño: None declared, Carmen Cobelo: None declared, Javier Sanchez Perez: None declared, Diego de Argila: None declared, Esteban Rubio-Romero: None declared, MANUEL LEON LUQUE: None declared, Juan María Blanco-Madrigal: None declared, E. Galindez: None declared, Javier Martin Ibanez: None declared, Santos Castañeda: None declared, Ricardo Blanco Speakers bureau: Abbvie, Pfizer, Roche, Bristol-Myers, Janssen and MSD, Consultant of: Abbvie, Pfizer, Roche, Bristol-Myers, Janssen and MSD, Grant/ research support from: Abbvie, MSD and Roche, Miguel A González-Gay Speak ers bureau: Pfizer, Abbvie, MSD, Grant/research support from: Pfizer, Abbvie, MSD, Raquel López-Mejías: None declared DOI: 10.1136/annrheumdis-2021-eular.766

\section{AB0097 \\ DIAGNOSTIC ACCURACY OF SERUM MARKERS IN LARGE VESSEL VASCULITIS AND CORRELATION WITH PET IMAGING}

R. Bilici Salman ${ }^{1}$, H. Satış2, A. Avanoglu Guler3, H. Karadeniz3, H. Küçük', S. Haznedaroglu ${ }^{1}$, A. Tufan ${ }^{1}$, B. Goker ${ }^{1}$, U. O. Akdemir ${ }^{4}$, L. O. Atay ${ }^{4}$, H. Paşaoğlu ${ }^{5}$, M. A. Ozturk' ${ }^{1}{ }^{1}$ Gazi University School of Medicine, Rheumatology, Ankara, Turkey; ${ }^{1}$ Gazi University School of Medicine, Rheumatology, Ankara, Turkey; ${ }^{1}$ Gazi University School of Medicine, Rheumatology, Ankara, Turkey; ${ }^{4}$ Gazi University School of Medicine, Nuclear Medicine, Ankara, Turkey; ${ }^{5}$ Gazi University School of Medicine, Biochemistry, Ankara, Turkey

Background: The onset of symptoms in large-vessel vasculitis (LVV) tends to be subacute, which often leads to a delay in diagnosis, during which time vascular disease may start and progress to become symptomatic. PET/CT has been recognised since the 2003s as a promising tool in evaluating of LVV. There is no gold standard diagnostic laboratory tests for this patient group, novel markers for active LVV is needed.

Objectives: to investigate the association between vascular inflammation, as detected by PET imaging and interleukin-6 (IL-6), pentraxin3 (PTX3), and B-cell-activating-factor (BAFF) in subjects with LVV.

Methods: The study included 67 patients patients with newly diagnosed GCA $(n=27)$ or TA $(n=9)$ and healthy control $(n=31)$ who had been referred to the Rheumatology Unit at Gazi University, between December 2017 and August 2020. PET images obtained from an 29 patients (22 with GCA and 7 with TA) who had not received any corticosteroid treatment prior to PET imaging and blood sampling. IL-6, PTX3, and BAFF levels were determined quantitatively by enzyme-linked immunosorbent assay (ELISA) kits.

Results: 36 patients with LVV (20 females, 16 males; age $64,5 \pm 16,6)$ and $31 \mathrm{HC}$ (14 females, 17 males; age37,1 $\pm 9,6$ ) were analysed. Serum levels of IL-6,PTX3, BAFF, ESR and CRP are increased in patients with newly diagnosed LVV compared with those in control subjects. In a ROC analysis, serum IL-6 provided excellent discrimination of newly diagnosed LVV patients from $\mathrm{HC}$, as indicated by AUCs $>0.90$. Serum BAFF also accurately distinguished newly diagnosed LVV patients from $\mathrm{HC}$ with AUCs $>0.80$. Serum PTX3 did not provide an AUC $>0.80$. In this study, we correlate vascular inflammation, as detected by PET imaging in newly diagnosed LVV patients, with the ESR, CRP. PTX3, IL-6 and BAFF. As a result, none of these markers has been associated with vascular inflammation as measured using PET.

Conclusion: In conclusion, our study shows that serum levels of PTX3, IL- 6 and BAFF are increased in most LVV patients. The diagnostic value of BAFF and IL-6, both separately and in combination, should be further evaluated in larger cohorts of LVV patients, as well as in patients with infections or other inflammatory conditions. However, none of these markers has been associated with vascular inflammation as measured using PET.

Disclosure of Interests: None declared DOI: 10.1136/annrheumdis-2021-eular.3719 